\title{
Die Trias von verfassungsorientierter und verfassungskonformer Auslegung sowie der verfassungskonformen Rechtsfortbildung - Ein Beitrag zu mehr Methodenehrlichkeit beim Streit zwischen den beiden Senaten des BVerfG
}

\author{
Thomas M.J. Möllers
}

\section{Einleitung}

\section{Pflicht zur verfassungskonformen Auslegung}

Die Verfassungswidrigkeit und Nichtigkeit einer Norm ist ultima ratio, also letztes Mittel. Die verfassungskonforme Auslegung ist damit im Zweifel der Nichtigkeit vorzuziehen. ${ }^{1}$ Mit anderen Worten: Sie führt nicht zur förmlichen Normverwerfung, sondern zur Normerhaltung. ${ }^{2}$ Die Pflicht zur verfassungskonformen Auslegung lässt sich mit verschiedenen Überlegungen begründen. Zum einen ist die Verfassung nach dem Stufenbau des Rechts höherrangig gegenüber dem einfachen Recht und schon wegen der lex-superior-Regel $\mathrm{zu}$ beachten. ${ }^{3}$ Zudem verlangen der Gedanke der

1 S. ständige Rechtsprechung, BVerfG, Beschl. v. 19.9.2007 (Az. 2 BvF 3/02), BVerfGE 119, 247, 274 - Teilzeitbeschäftigung von Beamten; BVerfG, Beschl. v. 14.10.2008 (Az. 1 BvR 2310/06), BVerfGE 122, 39, 60 f. - Beratungshilfe; BVerfG, Urt. v. 4.5.2011 (Az. 2 BvR 2365/09 u.a.), BVerfGE 128, 326, 400 - Sicherungsverwahrung. S. vorher bereits BVerfG, Beschl. v. 7.5.1953 (Az. 1 BvL 104/52), BVerfGE 2, 266, 282; BVerfG, Besch. v. 1.3.1978 (Az. 1 BvL 20/77), BVerfGE 48, 40, 45 f.; BVerfG, Beschl. v. 19.6.1979 (Az. 2 BvL 14/75), BVerfGE 51, 304, 323 - Jugendgefährdende Schriften; BVerfG, Beschl. v. 3.6.1992 (Az. 2 BvR 1041/88, 78/89), BVerfGE 86, 288, $320-\S 57$ a StGB.

2 Dreier, in: Dreier, GG, 3. Aufl. 2013, Art. 1 III Rn. 85.

3 Kelsen, Reine Rechtslehre, 2. Aufl. 1960, S. 228 ff.; Röhl/Röhl, Allgemeine Rechtslehre, 3. Aufl. 2008, S. 308 f.; Rüthers/Fischer/Birk, Rechtstheorie, 8. Aufl. 2015, Rn. 272 ff.; Bydlinski, Juristische Methodenlehre und Rechtsbegriff, 2. Aufl. 1991, S. 201; Kramer, Juristische Methodenlehre, 4. Aufl. 2013, S. 92. A.A. etwa Teubner, Soziale Systeme 2 (1996), 229, 231: „grandiose Selbsttäuschung des Rechts“. Zudem wird das Argument der Einheit der Rechtsordnung angebracht, s. Hesse, Grundzüge des Verfassungsrechts der Bundesrepublik Deutschland, 20. Aufl. 1995, Rn. 80. 
Rechtskontinuität und die Vermeidung eines Rechtsvakuums, die Norm im Zweifel aufrecht zu erhalten. Darüber hinaus fordert die Rechtsstaatlichkeit, dass auch unter den Staatsgewalten wechselseitig ein Übermaßverbot besteht. ${ }^{4}$ Letztlich gebietet der Respekt vor der gesetzgebenden Gewalt, ein Gesetz nicht für nichtig zu erklären, sondern zu erhalten, indem man es verfassungskonform auslegt. ${ }^{5}$ Es besteht somit ein Gebot zur Erhaltung der Norm (favor legis). ${ }^{6}$ Ähnlich besteht auch im anglo-amerikanischen Recht die Pflicht, die Norm so auszulegen, dass sie nicht gegen die Verfassung verstößt. ${ }^{7}$ Weniger überzeugend ist dagegen die Behauptung, es bestünde eine grundsätzliche Vermutung, dass ein Gesetz verfassungsmäßig sei. ${ }^{8}$ Dabei handelt es sich um einen klassischen Zirkelschluss (petitio principii), da das BVerfG gerade dazu da ist, zu prüfen, ob das Gesetz gegen das Grundgesetz verstößt. ${ }^{9}$

\section{Umstrittene Argumentationsfiguren des Verfassungsrechts}

a) Die unpräzise Verwendung der verfassungskonformen Auslegung durch das BVerfG

Die Pflicht zur verfassungskonformen Auslegung ist zwar seit vielen Jahren anerkannt, ansonsten wird dieser Begriff aber für vier verschiedene

4 Zippelius, in: FG 25 Jahre BVerfG, Bd. II, 1976, S. 108, 111.

5 Ständige Rechtsprechung (vgl. Fn. 1); Canaris, in: FS Kramer, 2004, S. 141, 147 ff.

6 Bogs, Die verfassungskonforme Auslegung von Gesetzen, 1966, S. 21 f.; Schlaich/ Korioth, Das Bundesverfassungsgericht, 10. Aufl. 2015, Rn. 442; Canaris, in: FS Kramer, 2004, S. 141, 149; Dannecker, in: LK-StGB, 12. Aufl. 2007, § 1 Rn. 326.

7 Gesetze sollen so ausgelegt werden, dass sie nicht gegen die Verfassung verstoßen, ebenso im US-amerikanischen Recht, Crowell v. Benson, 285 U.S. 22, 62 (1932): "When the validity of an act of the Congress is drawn in question, and even if a serious doubt of constitutionality is raised, it is a cardinal principle that this Court will first ascertain whether a construction of the statute is fairly possible by which the question may be avoided."; Posner, Statutory Interpretation, 50 U.Chi.L.Rev. 800, 815 (1983).

8 BVerfG, Beschl. v. 7.5.1953 (Az. 1 BvL 104/52), BVerfGE 2, 266, 282 - Notaufnahme.

9 Kritisch gegen eine solche Fiktion folglich Göldner, Verfassungsprinzip und Privatrechtsnorm in der verfassungskonformen Auslegung und Rechtsfortbildung, 1969, S. 45; Bettermann, Die verfassungskonforme Auslegung, 1986, S. 24 f.; Auer, in: Neuner, Grundrechte und Privatrecht aus rechtsvergleichender Sicht, 2007, S. 27, $32 \mathrm{f}$. 
Sachverhalte verwendet, die streng genommen unter diesen Begriff nicht passen: So betreibt das BVerfG in zahlreichen Entscheidungen verfassungskonforme Rechtsfortbildung, ohne diesen Begriff zu verwenden. Stattdessen wird zum Teil von verfassungskonformer Interpretation gesprochen. ${ }^{10}$ Schließlich verwendet das BVerfG auch nicht den Begriff der verfassungsorientierten Auslegung, obwohl dieser seit langem in der Rechtsliteratur eingeführt ist. ${ }^{11}$ Stattdessen wird nur recht unpräzise von der Ausstrahlungswirkung gesprochen. ${ }^{12}$

\section{b) Das Fachgericht als Verfassungsgericht}

Wegen der Ausstrahlungswirkung der Grundrechte ${ }^{13}$ sind alle Fachgerichte aufgerufen, neben ihrem einschlägigen Fachgebiet gleichzeitig auch das Grundgesetz zu beachten, um verfassungswidrige Entscheidungen zu verhindern. Sie sind insoweit auch Verfassungsgericht, sodass es in Deutschland tausende „Verfassungsgerichte“ gibt. ${ }^{14}$ Die unscharfe Verwendung des Begriffs der verfassungskonformen Auslegung durch das BVerfG macht es für die Fachgerichte nicht leicht, mit der Verfassung zu arbeiten. Einerseits grenzt die verfassungskonforme Auslegung an die verfassungs-

10 Z.B. BVerfG, Beschl. v. 19.9.2007 (Az. 2 BvF 3/02), BVerfGE 119, 247, 274 Teilzeitbeschäftigung von Beamten.

11 S. etwa Dreier, Die Verwaltung 36 (2003), 105, 111 Fn. 43; verwendet wird der Begriff aber in der Rechtsprechungssammlung Bumke/Voßkuhle, Casebook Verfassungsrecht, 2015, Rn. 161, 669.

12 Lerche, in: FS Odersky, 1996, S. 215, 216 f., 223, 225 f.; Canaris, Grundrechte und Privatrecht, 1999, S. 30, 93 spricht von einer Verlegenheitslösung dieser bildhaften Wendung der Umgangssprache. Die Ausstrahlungswirkung wird despektierlich auch als „Scheinwerfertheorie“ bezeichnet, s. Friedrich, in: Umbach/ Clemens/Dollinger, BVerfGG - Mitarbeiterkommentar, 2. Aufl. 2005, S. 93, 104 Fn. 55.

13 BVerfG, Urt. v. 15.1.1958 (Az. 1 BvR 400/51), BVerfGE 7, 198, 206 f. - Lüth. Deutlich auch BVerfG, Beschl. v. 11.6.1991 (Az. 1 BvR 239/90), BVerfGE 84, 192, 195 - Offenbarung der Entmündigung: „Der Richter hat kraft Verfassungsgebots zu prüfen, ob von der Anwendung zivilrechtlicher Vorschriften im Einzelfall Grundrechte berührt werden. Trifft das zu, dann hat er diese Vorschriften im Lichte der Grundrechte auszulegen und anzuwenden." S. auch BVerfG, Beschl.v. 19.10.1993 (Az. 1 BvR 567, 1044/89), BVerfGE 89, 213, 229 f. - Bürgschaft vermögensloser Angehöriger.

14 Schlaich/Korioth, Das Bundesverfassungsgericht, 10. Aufl. 2015, Rn. 441: ,[...] die verfassungskonforme Auslegung einem jeden Richter obliegt.“ 
orientierte Auslegung, die keinen Vorrang hat, sondern nur einen Abwägungsmaßstab darstellt. ${ }^{15}$ Andererseits ist wenig geklärt, wann eine verfassungskonforme Auslegung unzulässig ist und in welchem Umfang die verfassungskonforme Rechtsfortbildung noch möglich ist. ${ }^{16}$ Damit besteht die Gefahr, dass die Fachgerichte mit dieser Aufgabe überfordert sind. ${ }^{17}$

c) Prüfung von Entscheidungen der Fachgerichte - zur Prüfungsintensität des BVerfG (,Superrevisionsinstanz“)

Mit einer Verfassungsbeschwerde kann jeder Bürger vor dem Bundesverfassungsgericht Verstöße gegen seine Grundrechte geltend machen, wenn der anderweitige Rechtsweg erschöpft ist. Gerichte sind gem. Art. 20 Abs. 3 GG an Recht und Gesetz und die Rechtsanwendungsgleichheit gebunden, weshalb grundsätzlich jedes fehlerhafte Urteil erfolgreich vor dem BVerfG geprüft werden kann.

Ausdrücklich versteht sich das BVerfG nicht als letztinstanzliches Gericht, das andere höchste Gerichte korrigiert. Es sieht sich nicht als „Superrevisionsinstanz“, sondern überprüft lediglich, ob „spezifisches Verfas-

15 S. unten IV. Von fließenden Grenzen spricht Kuhlen, Die verfassungskonforme Auslegung von Strafgesetzen, 2006, S. 3; s. auch Zippelius, Juristische Methodenlehre, 11. Aufl. 2012, § 7.g), S. 33.

16 S. unten III. Schulze-Fielitz, in: Dreier, GG, 3. Aufl. 2015, Art. 20 Rn. 87.

17 Schlaich/Korioth, Das Bundesverfassungsgericht, 10. Aufl. 2015, Rn. 145; Wieland, in: Dreier, GG, 2. Aufl. 2008, Art. 100 Rn. 19; Ruffert, JZ 2009, 389, 397 f. zur Beurteilung der Rechtsprechung des BAG. Beispielhaft ist Macrotron-Entscheidung des BGH. Dieser sah durch den Rückzug von der Börse die Verkehrsfähigkeit der Aktie und damit das Aktieneigentum im Sinne von Art. 14 Abs. 1 GG verletzt. Zum Schutz der Minderheitsaktionäre entwickelte er rechtsfortbildend die Voraussetzung, dass die Gesellschaft einen Beschluss der Hauptversammlung sowie ein Pflichtangebot der Aktiengesellschaft oder des Großaktionärs über den Kauf der Aktien der Minderheitsaktionäre herbeiführen müsse. Das BVerfG bejahte zwar grundsätzlich das Recht des BGH zur Rechtsfortbildung, lehnte es im konkreten Fall aber ab, die Rechtsfortbildung mit einer Verletzung des Eigentumsrechts zu begründen, da die Verkehrsfähigkeit der Aktie als bloßer Vermögenswert nicht unter den Schutzbereich des Eigentums gem. Art. 14 Abs. 1 GG falle. BGH, Urt. v. 25.11.2002 (Az. II ZR 133/01), BGHZ 153, 47, 55 - Macrotron; BVerfG, Urt. v. 11.7.2012 (Az. 1 BvR 3142/07, 1569/08), BVerfGE 132, 99 Rn. 54 ff. - MVS/Lindner. 
sungsrecht" verletzt wurde. ${ }^{18}$ Eine gewisse Zurückhaltung ist schon deshalb sinnvoll, weil Fachgerichte grundsätzlich in dem jeweiligen Fachgebiet naturgemäß über eine größere Sachnähe verfügen, den streitigen Sachverhalt zu entscheiden, als das Bundesverfassungsgericht. ${ }^{19}$ Vor allem wird die Verfassung überanstrengt: Würden alle Probleme auf die Verfassung abgeladen, geht das Gefühl verloren, welche Probleme wirklich wichtig sind und des Schutzes der Verfassung bedürfen. ${ }^{20}$ Deshalb sollte man mit dem Verfassungsrecht zurückhaltend verfahren, um Auslegungsergebnisse des einfachen Rechts korrigieren zu wollen. Denn in der Regel hat das einfache Recht die deutlich präziseren Normen, um die Interessen der Parteien zu einem Ausgleich zu bringen, ${ }^{21}$ während die Grundrechte als Prinzipien der weiteren Konkretisierung und oft einer umfangreichen Abwägung bedürfen.

Allerdings wird dem BVerfG vorgeworfen, dass es sich oft nicht an seine eigenen Vorgaben hält und mit der Verfassungsbeschwerde doch einfaches Recht prüft und damit eine „Banalisierung der Grundrechte“22 zulässt. So wird etwa die Eigenbedarfskündigung im Mietrecht weitgehend von der Rechtsprechung des BVerfG bestimmt. ${ }^{23}$

18 BVerfG, Beschl. v. 10.6.1964 (Az. 1 BvR 37/63), BVerfGE 18, 85, 92; BVerfG, Beschl. v. 19.10.1993 (Az. 1 BvR 567, 1044/89), BVerfGE 89, 214, 230 - Bürgschaft gegenüber Angehörigen; s. auch BVerfG, Urt. v. 11.7. 2012 (1 BvR 3142/07, 1569/08), NJW 2012, 3081 Rn. 76 - Delisting.

19 Neuner, in: Diederichsen/Sellert, Das BGB im Wandel der Epochen, 2002, S. 131, 150.

20 Deutlich Röhl/Röhl, Allgemeine Rechtslehre, 3. Aufl. 2008, § 84 II., S. 673.

21 Anschaulich Rüfner, HStR, Bd. IX, 3. Aufl. 2011, § 197 Rn. 112: „Die allgemein gefaßten Grundrechtsnormen vermögen die Feinarbeit des Zivilrechts und dessen Interpretation nicht zu ersetzen oder zu verdrängen."; s. auch Lerche, in: FS Odersky, 1996, S. 215, 232.

22 So die instruktiven Ausführungen des Richters Grimm, in: Sondervotum zu BVerfG, Beschl. v. 6.6.1989 (Az. 1 BvR 921/85), BVerfGE 80, 137, 164 ff. - Reiten im Walde.

23 Kritisch Palandt/Weidenkaff, BGB, 73. Aufl. 2014, §573 Rn. 23. Etwa als es das Besitzrecht des Mieters unter den Eigentumsschutz des Art. 14 Abs. 1 GG stellte, s. BVerfG, Beschl. v. 26.5.1993 (Az. 1 BvR 208/93), BVerfGE 89, 1, 6 ff. S. auch Ruffert, JZ 2009, 389, 394 f. Polemisch Sendler, NJW 1994, 1518, 1519: „oberstes Amtsgericht insbesondere für Eigenbedarfsklagen von Vermietern“. 
d) Die Dreiteilung von verfassungsorientierter und verfassungskonformer Auslegung sowie Rechtsfortbildung

Christoph Vedder hat 20 Jahre an der Juristischen Fakultät der Universität Augsburg gewirkt und das Verfassungsrecht, das Europarecht und das Völkerrecht beeinflusst. Deshalb dient der Beitrag dem intradisziplinären Austausch der sonst abgeschiedenen juristischen Spezialdisziplinen.

Nach der hier vertretenen Ansicht sollen drei methodische Figuren unterschieden werden, die das einfache Recht beeinflussen: die wenig geklärte verfassungsorientierte Auslegung (II.), die bekannte Figur der verfassungskonformen Auslegung (III.) und, davon abzugrenzen, die verfassungskonforme Rechtsfortbildung (IV.). Die Verfassung ist einerseits Auslegungsmaßstab für das einfache Recht, besteht aber andererseits selbst aus auslegungsfähigen und auslegungsbedürftigen Normen. ${ }^{24}$ Abgesehen von solchen paradoxen Erkenntnissen soll deutlich werden, dass die Verfassung ein abstraktes Fundament ist, das Spielraum bei der Auslegung der einfachen Gesetze lässt sowie erstens eine begrenzende Funktion hat (verfassungskonforme Auslegung), zweitens als ultima ratio Gestaltungsauftrag an die Rechtsprechung ist (verfassungskonforme Rechtsfortbildung) und drittens eine abwägende Rolle ohne Vorrang hat (verfassungsorientierte Auslegung).

\section{Die verfassungsorientierte Auslegung}

\section{Der Meinungsstreit zur verfassungsorientierten Auslegung}

Die verfassungsorientierte Auslegung gehört zu den am wenigsten geklärten Rechtsfiguren. Vom BVerfG wird die Rechtsfigur bis heute nicht ausdrücklich verwendet; stattdessen wird etwa von Ausstrahlungswirkung bzw. den Grundrechten als Werteordnung ${ }^{25}$ gesprochen. Nach einer Literaturansicht sei die Abgrenzung zwischen verfassungsorientierter und ver-

24 Zippelius, in: FG 25 Jahre BVerfG, Bd. II, 1976, S. 108, 112.

25 BVerfG, Urt. v. 15.1.1958 (Az. 1 BvR 400/51), BVerfGE 7, 198, 205 - Lüth (Fn. 13); BVerfG, Urt. v. 25.2.1975 (Az. 1 BvF 1/74 u.a.), BVerfGE 39, 1, 67 Schwangerschaftsabbruch I. 
fassungskonformer Auslegung unscharf. ${ }^{26}$ Manche gehen sogar so weit, dass verfassungsorientierte und verfassungskonforme Auslegung im Ergebnis eins seien. ${ }^{27}$ Die Figur der verfassungsorientierten Auslegung sei insoweit überflüssig. ${ }^{28}$ Nach einer zweiten Ansicht dürfe die verfassungsorientierte Auslegung von Jedermann, die verfassungskonforme Auslegung aber nur vom BVerfG durchgeführt werden. ${ }^{29}$ Dem ist entgegenzuhalten, dass eine Unterscheidung nach dem Adressaten verfassungsrechtlich nicht begründet werden kann. ${ }^{30}$ Nach einer dritten Ansicht dient die verfassungskonforme Auslegung in einem ersten Schritt der Ausscheidung solcher im Wege grammatikalischer, historischer, teleologischer Auslegung gefundener Ergebnisse, die mit der Verfassung nicht vereinbar sind. Die verfassungsorientierte Auslegung diene in einem zweiten Schritt dazu, aus den verfassungskonformen Ergebnissen dasjenige auszuwählen, das der Verfassung bzw. dem verfassungsrechtlichen Schutzauftrag am nächsten kommt. ${ }^{31}$ Damit bestünde eine Parallelität zum europarechtlichen ,effet utile".32

An dieser Ansicht gefällt, dass die verfassungsorientierte Auslegung die Grundrechte zur Abwägung heranzieht und insoweit wie Prinzipien verwendet. Allerdings ist sie zu eng, da sie in einem zweiten Schritt erst nach der verfassungskonformen Auslegung zur Anwendung kommen will. Die verfassungsorientierte Auslegung hat aber auch einen Anwendungsbereich, wenn die Verfassung gar nicht zu einem Vorrang zwingt, es also gar keine verfassungswidrigen Ergebnisse gibt. Zudem kann das Optimie-

26 Dannecker, in: LK-StGB, 12. Aufl. 2007, § 1 Rn. 333; Kuhlen, Die verfassungskonforme Auslegung von Strafgesetzen, 2006, S. 2: „Grenzen fließend“.

27 Lembke, Einheit aus Erkenntnis?, 2009, S. 247; Reimer, Juristische Methodenlehre, 2016, Rn. $400 \mathrm{f}$.

28 Etwa Canaris, in: FS Kramer, 2004, S. 141, 154.

29 Simon, EuGRZ 1974, 85, 87; Stern, Das Staatsrecht der Bundesrepublik Deutschland, Bd. I, 2. Aufl. 1984, S. 136.

30 Wendt, in: FS Würtenberger, 2013, S. 123, 126.

31 Wendt, in: FS Würtenberger, 2013, S. 123, 130.

32 Armbrüster, NJW 2007, 1494, 1496. Zum ,effet utile“ etwa EuGH, Urt. v. 15.7.1963, Rs. 34/62, ECLI:EU:C:1963:18 = Slg. 1963, 269, 318 - Deutschland/ Kommission; Kutscher, in: Gerichtshof der Europäischen Gemeinschaften, Begegnung von Justiz und Hochschule, 1976, S. I-43; Hager, Rechtsmethoden in Europa, 2009, S. 254; Pechstein/Drechsler, in: Riesenhuber, Europäische Methodenlehre, 2. Aufl. 2010, § 8 Rn. 55 ff.; Everling, JZ 2000, 217, 223. 
rungsgebot schon Teil der verfassungskonformen Auslegung sein. ${ }^{33}$ Damit ist die verfassungskonforme Auslegung der verfassungsorientierten Auslegung nachrangig. ${ }^{34}$

\section{Begriff der verfassungsorientierten Auslegung}

Nach der hier vertretenen Ansicht liegt eine verfassungsorientierte Auslegung vor, wenn die Grundrechte nur eine einfache Argumentationsfigur darstellen, welche gegenüber den bisherigen Auslegungsfiguren (nur) gleichrangig ist. Der Einfluss der Verfassung auf das einfache Recht ist damit so schwach, dass es gerade keinen Vorrang der Verfassung verlangt, sondern sich im Zweifel gegenüber den anderen Figuren nicht durchsetzt. Hier soll nur die Ausstrahlungswirkung der Grundrechte im Zivilrecht und im Strafrecht aufgezeigt werden. Damit ist eine Abgrenzung zwischen verfassungsorientierter und verfassungskonformer Auslegung in dreierlei Hinsicht möglich:

Erstens verlangt die verfassungsorientierte nur, die Verfassung zu berücksichtigen und in die Abwägung einzubeziehen. ${ }^{35}$ Die verfassungskonforme Auslegung ist stärker: Sie zwingt den Rechtsanwender bei verschiedenen möglichen Auslegungsergebnissen dazu, das verfassungskonforme Ergebnis den anderen vorzuziehen.

Folglich ist zweitens die verfassungsorientierte Auslegung nur ein Kriterium unter mehreren Interpretationsfiguren. Sie ist insoweit nur eine gleichberechtige Auslegungsfigur, ohne einen Vorrang zu fordern, ${ }^{36}$ während umgekehrt die verfassungskonforme Auslegung diesen Vorrang gerade erzwingt, indem sie verfassungswidrige Ergebnisse aussondert. Sie ist damit nur eine Abwägungsregel, ohne irgendeine Vermutungswirkung. Kudlich beschreibt hierzu sehr plastisch einen Wendepunkt zwischen verfassungsorientierter und verfassungskonformer Auslegung: „(...) in dem

33 Reimers, Juristische Methodenlehre, 2016, Rn. 400. A.A. Wendt, in: FS Würtenberger, 2013, S. 123, 130.

34 Höpfner, Die systemkonforme Auslegung, 2008, S. 180.

35 Burmeister, Verfassungsorientierung, 1966, S. 14 ff.; Simon, EuGRZ 1974, 85, 86 f.; Stern, Das Staatsrecht der Bundesrepublik Deutschland, Bd. 1, 2. Aufl. 1984, S. 136; Schlaich/Korioth, Das Bundesverfassungsgericht, 10. Aufl. 2015, Rn. 448; Kudlich, JZ 2003, 127, 130.

36 Höpfner, Die systemkonforme Auslegung, 2008, S. 180; in Ansätzen bereits Skouris, Teilnichtigkeit von Gesetzen, 1973, S. 115. 
Moment, in dem die Interpretation dann verfassunsgswidrig wird, schlägt die Argumentation in eine verfassungskonforme Auslegung um, die zumindest ein bestimmtes Ergebnis zwingend verbietet. " 37 Während die verfassungskonforme Auslegung als Regel bezeichnet werden kann, ist die verfassungsorientierte Auslegung insoweit nur ein Prinzip, ${ }^{38}$ welches bei der Abwägung zu berücksichtigen ist. ${ }^{39}$ So können Grundrechtspositionen zur Verteidigung gegen die Lösung des einfachen Rechts in die Argumentation einbezogen werden, ohne dass sie sich letztlich durchsetzen. Andererseits können sie auf beiden Seiten betroffen sein und in die Abwägung einbezogen werden. Es geht typischerweise um die Ausstrahlungswirkung, ${ }^{40}$ also die Grundrechtsentfaltung und Grundrechtsdurchsetzung. Typische Anwendungfälle sind etwa die Meinungs-, Koalitions- und Berufsfreiheit, die im Arbeitsrecht zu berücksichtigen sind.

Und drittens zielt die verfassungskonforme Auslegung auf Normerhaltung, ${ }^{41}$ während die verfassungsorientierte Auslegung eine Normoptimierung ${ }^{42}$ bezweckt, ohne dass aber ansonsten die Verfassungswidrigkeit der Norm droht. Normoptimierung ist demnach so zu verstehen, dass neben den üblichen Auslegungskriterien noch weitere - verfassungsrechtliche Aspekte in die Waagschale geworfen werden, welche im Ergebnis aber nicht zwingend überwiegen müssen.

3. Die verfassungsorientierte Auslegung als einfache Abwägungsregel im Zivilrecht

a) Eigentumsfreiheit des Vermieters v. Rechte des Mieters

Nach früherem Recht konnte beim Tod des Mieters nur der Ehegatte oder Familienangehörige in den Mietvertrag eintreten, § 569a BGB a.F. (jetzt:

37 Kudlich, JZ 2003, 127, 130 Fn. 33.

$38 \mathrm{Zu}$ den Begriffen Regel und Prinzip s. Dworkin, Taking Rights Seriously, 2. Aufl. 1978, S. 24 f. (deutsch: Bürgerrechte ernst genommen, 1984); Alexy, Theorie der Grundrechte, 1986, S. 76; (engl.: Theory of Constitutional Rights, 2002); ders., ARSP Beiheft 25 (1985), 13, 20.

39 Dannecker, in: LK-StGB, 12. Aufl. 2007, § 1 Rn. 332; Kuhlen, Die verfassungskonforme Auslegung von Strafgesetzen, 2006, S. 2.

40 S. oben Fn. 13.

41 Dreier, in: Dreier, GG, 3. Aufl. 2013, Art. 1 III Rn. 85 ff.

42 Reimer, Juristische Methodenlehre, 2016, Rn. 398. 
$\S 563$ BGB). Das BVerfG billigte die Rechtsfortbildung des BGH, § 569a BGB analog auf die nichteheliche Lebensgemeinschaft anzuwenden, unter anderem auch deshalb, weil der Gesetzgeber und die Rechtsprechung die nichteheliche Lebensgemeinschaft schon vorher in verschiedenen Gesetzen anerkannt hatten. ${ }^{43}$

Dies ist von der Literatur massiv kritisiert worden, u.a. mit dem Argument, die über die allgemeine Handlungsfreiheit nach Art. 2 Abs. 1 GG geschützte Vertragsfreiheit und das Eigentumsrecht des Art. 14 GG würden dadurch in unzulässiger Weise verletzt. ${ }^{44}$ Dem ist entgegenzuhalten, dass der Gesetzgeber die Vertragsfreiheit und das Eigentumsrecht des Vermieters in den letzten Jahrzehnten bereits umfangreich eingeschränkt hat. ${ }^{45}$ Hintergrund ist die Überlegung, dass der Mieter in seinem sozialen Umfeld einen besonderen Schutz erfahren soll. ${ }^{46}$ Insoweit ist es in sich schlüssig, mit einem Ähnlichkeitsvergleich die nichteheliche Lebensgemeinschaft daran teilhaben zu lassen. Im Ergebnis führen die Grundrechte also nicht zu einem Vorrang.

Im Fall des Eintrittsrechts der nichehelichen Lebensgemeinschaft in den Mietvertrag kann sich der Vermieter zwar auf das Eigentumsrecht des Art. 14 GG berufen. Die Rechte des Vermieters aus Art. 14 GG wurden zwar in die Waagschale gelegt, sie setzen sich aber gerade nicht durch und sind damit nur eine Abwägungsposition. Das ist der typische Fall der verfassungsorientierten Auslegung.

b) Eingriff in Unternehmen versus Meinungsfreiheit

aa) Im Lüth-Urteil entwickelte das BVerfG den Einfluss der Verfassung auf das Zivilrecht (das „Ob“). Ein Boykottaufruf wurde bisher als sittenwidrige Schädigung nach $\S 826$ BGB bzw. als Eingriff in den eingerichteten und ausgeübten Gewerbebetrieb gem. § 823 Abs. 1 BGB durch die Zivilgerichte eingestuft. Allerdings kann der Boykottaufruf in den Schutzbereich der Meinungsfreiheit gem. Art. 5 Abs. 1 GG fallen. § 826 BGB darf somit nicht nur nach den Wertungen des BGB ausgelegt werden (so dass

43 BGH, Urt. v. 13.1 .1993 (Az. 8 ARZ 6/92), BGHZ 121, 116, 121 ff. - § 569a analog; BVerfG, Beschl. v. 3.4.1990 (Az. 1 BvR 1186/8), BVerfGE 82, 6, 15 f. Nichteheliche Lebensgemeinschaft und Mietnachfolge.

44 Roellecke, JZ 1990, 813; Hillgruber, JZ 1996, 118, 119, 122; Diederichsen, Jura 1997, 57, 62 ff.; Rüthers/Fischer/Birk, Rechtstheorie, 8. Aufl. 2015, Rn. 875.

45 Zur Rechtsprechung s. unten Fn. $63 \mathrm{ff}$.

46 BVerfG, Beschl. v. 3.4.1990 (Az. 1 BvR 1186/8), BVerfGE 82, 6, 16 f. 
jeder Boykottaufruf rechtswidrig wäre), sondern muss auch das Grundgesetz als Werteordnung und die Ausstrahlungswirkung der Grundrechte, hier der Meinungsfreiheit, berücksichtigen. Die verfassungskonforme Auslegung zwingt dazu, die Rechtswidrigkeit des Boykottaufrufs zu verneinen und diesen im Lichte der Verfassung auszulegen. Hier scheint das Bild von der mittelbaren Drittwirkung überzeugend. Das BVerfG nahm nicht sofort eine Güterabwägung vor, sondern konkretisierte die Kollision zwischen Meinungsfreiheit (Art. 5 GG) einerseits sowie der Berufs- (Art. 12 GG) und Eigentumsfreiheit (Art. 14 GG) andererseits weiter. Maßgeblich sind Motive, Ziel und Zweck der Meinungsäußerung: Wenn es sich um einen Beitrag zum geistigen Meinungskampf in einer die Öffentlichkeit wesentlich berührenden Frage handelt, gelte die Vermutung für die Zulässigkeit der freien Rede. ${ }^{47}$ Dann schlägt die verfassungsorientierte in eine verfassungskonforme Auslegung um, weil sich das eine Grundrecht durchsetzt und das einfachgesetzliche Ergebnis korrigiert. Im Sinne der Verhältnismäßigkeit wird man zudem fordern müssen, dass der Boykottaufrufer vor einem Boykottaufruf den Dialog mit dem Boykottierten suchen muss. ${ }^{48}$

bb) Ein Grundrecht kann aber auch nur eine einfache Abwägungsregel sein, ohne das Ergebnis bereits zu präjudizieren. Dient der Boykottaufruf dagegen dazu, über eine bestimmte Meinungsgrundlage hinaus in den individuellen Bereich des wirtschaftlichen Wettbewerbs bestimmter Marktkonkurrenten einzugreifen, indem etwa wirtschaftliche Nachteile bei Nichtbefolgung angedroht werden ${ }^{49}$ so wird das Recht auf freie Meinungsäußerung und das Informationsinteresse der Allgemeinheit lediglich als Mittel zum Zweck der Förderung privater Wettbewerbsinteressen eingesetzt. In diesem Fall wird ein prinzipieller Vorrang der Interessen des Boykottierten angenommen. ${ }^{50}$ Die Meinungsfreiheit desjenigen, der zum Boykott aufruft, ist nur ein Argument unter mehreren; es setzt sich nicht durch und ist insoweit auch nicht vorrangig. Auch eine verfassungskon-

47 BVerfG, Urt. v. 15.1.1958 (Az. 1 BvR 400/51), BVerfGE 7, 198, 216 ff. - Lüth; BGH, Urt. v. 21.6.1966 (Az. VI ZR 261/64), BGHZ 45, 296, 308 - Höllenfeuer.

48 Möllers, NJW 1996, 1374, 1376 f.

49 BVerfG, Urt. v. 26.2.1969 (Az. 1 BvR 619/63), BVerfGE 25, 256, 264 f. - Blinkfüer.

50 BGH, Urt. v. 2.2.1984 (Az. I ZR 4/82), NJW 1985, 60, 62 Rn. 38 - Röster Uhren. 
forme Auslegung ist hier nicht einschlägig. ${ }^{51}$ Es bleibt bei der verfassungsorientierten Auslegung.

\section{Verfassungskonforme Auslegung}

\section{Grundsatzfragen zur verfassungskonformen Auslegung}

a) Die Begrifflichkeit nach den Vorgaben des Bundesverfassungsgerichts

Eine Norm ist verfassungsgemäß auszulegen, wenn von mehreren Auslegungsmöglichkeiten nur eine verfassungsgemäß ist. In den meisten Fällen dürfte es aber mehrere verfassungsmäßige Auslegungen geben. Umgekehrt ist es damit verboten, ein verfassungswidriges Auslegungsergebnis zu wählen. ${ }^{52}$ Die verfassungskonforme Auslegung übernimmt folglich eine Filterfunktion. Die Verfassung ist insoweit nicht nur Erkenntnis-, sondern zugleich auch Kontrollnorm. ${ }^{53}$ Bleibt nach der verfassungskonformen Auslegung kein verfassungskonformes Auslegungsergebnis übrig, ist die Vorschrift grundsätzlich als verfassungswidrig zu verwerfen, es sei denn, es bleibt Raum für eine verfassungskonforme Rechtsfortbildung. Nach Ansicht des BVerfG soll die Grenze der zulässigen verfassungskonformen Auslegung erreicht sein, wenn einer Auslegung der Gesetzeswortlaut und der Zweck, den der Gesetzgeber mit der Norm eindeutig verfolgen wollte, entgegensprechen. ${ }^{54}$ Die verfassungskonforme Auslegung bewegt sich innerhalb der Wortlautgrenzen und tritt typischerweise bei Generalklauseln oder unbestimmten Rechtsbegriffen auf.

51 A.A. Wendt, in: FS Würtenberger, 2013, S. 123, 130, wonach die verfassungsorientierte Auslegung der verfassungskonformen Auslegung nachfolge.

52 Bogs, Die verfassungskonforme Auslegung von Gesetzen, 1966, S. 94 f.; Zippelius, in: FG 25 Jahre BVerfG, Bd. II, 1976, S. 108, 111.

53 Wank, Grenzen richterlicher Rechtsfortbildung, 1978, S. 97 ff.; Voßkuhle, AöR 125 (2000), 177, 181.

54 S. etwa BVerfG, Beschl. v. 19.9.2007 (Az. 2 BvF 3/02), BVerfGE 119, 247, 274 Teilzeitbeschäftigung von Beamten. 
b) Vorrang der Verfassung als eigenständige Auslegungsfigur

Die verfassungskonforme Auslegung wird überwiegend zur systematischen Auslegung gezählt, ${ }^{55}$ andere sprechen von einer Variante der systematisch-teleologischen Interpretation. ${ }^{56}$ Das ist insoweit richtig, als man den Stufenbau des Rechts als Teil des äußeren Systems verstehen kann. Während die teleologische Auslegung in der Regel den Zweck einer Norm innerhalb der Kodifikation oder zumindest innerhalb des Rechtsgebietes, wie etwa dem Zivilrecht verortet, erweitert die verfassungskonforme Auslegung eben diesen Blick, indem sie auch die Stellung der Vorschrift im Gesamtgefüge der Normenhierarchie und die übergeordnete Stellung der Verfassung in die Auslegung einfließen lässt.

Allerdings stellt die Verfassung eine eigene Werteordnung ${ }^{57}$ dar, die über den Prinzipien des BGB und StGB steht und Auslegungsergebnisse, die lediglich auf Grundlage des einfachen Rechts gefunden wurden, korrigieren kann. Diese Ansicht berücksichtigt zu wenig, dass die Verfassung zwar einerseits Abwägungsmaßstab sein kann, andererseits aber dem einfachen Recht auch zwingend vorgehen kann, weil bei mehreren Auslegungsergebnissen die verfassungskonforme Auslegung vorzugehen hat. ${ }^{58}$ Insoweit liegt eine Vorrangregel vor. ${ }^{59}$ Die verfassungskonforme Ausle-

55 Müller/Christensen, Juristische Methodik I, 11. Aufl. 2013, Rn. 100; Röhl/Röhl, Allgemeine Rechtslehre, 3. Aufl. 2008, S. 623; Raisch, Juristische Methoden, 1995, S. 180; Rüthers/Fischer/Birk, Rechtstheorie, 7. Aufl. 2013, Rn. 759, anders dann aber in Rn 762a: "systemkonforme Rechtsanwendung"; Palandt/Sprau, BGB, 76. Aufl. 2017, Einl. Rn. 42.

56 Bydlinski, Juristische Methodenlehre und Rechtsbegriff, 2. Aufl. 1991, S. 455.

57 BVerfG, Urt. v. 15.1.1958 (Az. 1 BvR 400/51), BVerfGE 7, 198, 205 - Lüth; BVerfG, Urt. v. 25.2.1975 (Az. 1 BvF 1/74 u.a.), BVerfGE 39, 1, 67 - Schwangerschaftsabbruch I.

58 S. ständige Rechtsprechung, BVerfG, Beschl. v. 19.9.2007 (Az. 2 BvF 3/02), BVerfGE 119, 247, 274 - Teilzeitbeschäftigung von Beamten; BVerfG, Beschl. v. 14.10.2008 (Az. 1 BvR 2310/06), BVerfGE 122, 39, 60 f. - Beratungshilfe; BVerfG, Urt. v. 4.5.2011 (Az. 2 BvR 2365/09 u.a.), BVerfGE 128, 326, 400 - Sicherungsverwahrung. S. vorher bereits BVerfG, Beschl. v. 7.5.1953 (Az. 1 BvL 104/52), BVerfGE 2, 266, 282; BVerfG, Besch. v. 1.3.1978 (Az. 1 BvL 20/77), BVerfGE 48, 40, 45 f.; BVerfG, Beschl. v. 19.6.1979 (Az. 2 BvL 14/75), BVerfGE 51, 304, 323 - Jugendgefährdende Schriften; BVerfG, Beschl. v. 3.6.1992 (Az. 2 BvR 1041/88, 78/89), BVerfGE 86, 288, 320 - § 57a StGB.

59 Canaris, in: FS Kramer, 2004, 141, 143 ff.; Höpfner, Die systemkonforme Auslegung, 2008, S. 183; Reimer, Juristische Methodenlehre, 2016, Rn. 632. 
gung ist folgerichtig eine eigene Auslegungsfigur. ${ }^{60}$ Erforderlich ist eine zweistufige Prüfung: Nach dem Ergebnis des einfachen Rechts ist die verfassungsrechtliche Komponente in einem zweiten Schritt zu berücksichtigen.

\section{Verfassungskonforme Auslegung im Zivilrecht}

Exemplarisch sollen zwei Teilbereiche des Zivilrechts angeführt werden, in denen die verfassungskonforme Auslegung von der Rechtsprechung als Instrument herangezogen wurde. Einerseits handelt es sich dabei um die Begründung von Schutzpflichten, insbesondere zum Schutz der Gesundheit und des allgemeinen Persönlichkeitsrechts (a), andererseits wird die Selbstbestimmtheit durch eingeschränkte Zulässigkeit vertraglich auferlegter Beschränkung geschützt (b).

a) Schutz von Rechtsgütern und des allgemeinen Persönlichkeitsrechts

Eine ausdrückliche gesetzliche Grundlage für die Selbstbestimmungsaufklärung des Patienten bei Heileingriffen fehlte bisher. Der BGH erachtete einen Heileingriff immer für rechtswidrig, solange der Patient nicht aufgeklärt wurde und wirksam eingewilligt hatte. ${ }^{61}$ Mit dieser verfassungskonformen Auslegung des Tatbestandsmerkmals „Rechtswidrigkeit“ bei $\S 823$ Abs. 1 BGB konnte er somit eine Aufklärung des Arztes erzwingen. Das Erfordernis der informierten Einwilligung zu diagnostischen, vorbeugenden und heilenden Eingriffen folgt aus dem im Grundgesetz verankerten Recht auf körperliche Unversehrtheit (Art. 2 Abs. 2 GG) und aus der Freiheit und Würde der menschlichen Persönlichkeit (Art. 1 und 2 Abs. 1 GG). ${ }^{62}$ Inzwischen hat der Gesetzgeber diese Rechtsprechung in den $\S 630 \mathrm{~d}$ und $\S 630 \mathrm{e}$ BGB normiert.

60 Auer, in: Neuner, Grundrechte und Privatrecht aus rechtsvergleichender Sicht, 2007, S. 27, 31; Reimer, Juristische Methodenlehre, 2016, Rn. 630.

61 RG 31.5.1894 - 1406/94.; RG 27.5.1908 - VI 484/07; BGH 9.12.1958 - VI ZR 203/57 - Elektroschock II; BGH 14.2.1989 - VI ZR 65/88 - Arzthaftung für nicht aufklärungsbedürftiges Eingriffsrisiko; BGH 28.11.1957 - 4 StR 525/57 - Myom.

62 S. das Minderheitsvotum, in: BVerfG, Beschl. v. 25.7.1979 (Az. 2 BvR 878/74), BVerfGE 52, 131, 173 - Arzthaftungsprozess; a.A. das Mehrheitsvotum in BVerfGE (a.a.O., 131, 168 f). 
b) Abwehr von übermäßigen Freiheitsbeschränkungen

In diesen Fällen geht es weniger um die verfassungskonforme Auslegung einer Norm als um die Beschränkung vertraglicher Vereinbarungen. Die Rechtsprechung schränkt Vertragsabreden ein, wenn ein Vertragspartner übermäßig in seiner Freiheit eingeschränkt wird. Unzulässig, d.h. sittenwidrig gem. § $138 \mathrm{BGB}$, sind etwa die vertraglich vereinbarte Festlegung des Wohnsitzes ${ }^{63}$ oder die Pflicht zur Einnahme empfängnisverhütender Mittel. ${ }^{64}$ Unzulässig ist auch die Ausnutzung von Unterlegenheit, so dass eine Seite nicht selbstbestimmt handeln kann. ${ }^{65}$ Bei einem Ehevertrag ist dies zu bejahen, wenn eine unverheiratete schwangere Frau sich vor die Alternative gestellt sieht, in Zukunft entweder für das noch ungeborene Kind alleine sorgen zu müssen oder einen Ehevertrag abzuschließen, der für den Fall der Scheidung Unterhaltsansprüche vertraglich ausschließt. ${ }^{66}$ Eine übermäßige Freiheitsbeschränkung liegt aber auch dann vor, wenn eine Seite finanziell stark überfordert wird.

Bürgschaft vermögensloser Angehöriger: Eine 21-jährige Tochter, die keine Berufsausbildung hatte, überwiegend arbeitslos war und zur Zeit der Bürgschaftserklärung in einer Fischfabrik 1.150,- DM netto pro Monat verdiente, bürgte für ihren Vater für einen Kredit in Höhe von 100.000,- DM. Bis in die 90er Jahre des letzten Jahrhunderts hatte die Rechtsprechung die Auffassung vertreten, dass junge Familienangehörige, die volljährig sind, sich jederzeit unbegrenzt verbürgen können, denn grundsätzlich könne ein Volljähriger das Risiko abschätzen. Die Bürgschaft sei nun einmal ein riskantes Geschäft und ausdrücklich vom BGB zugelassen. Die Bindung an Recht und Gesetz nach Art. 20 Abs. 3 GG verbiete es, riskante Geschäfte, deren Erwartungen und Hoffnungen sich im Nachhinein für den Schuldner nicht erfüllen, als unklug aufzufassen, mit der Folge, dass eine Freistellung von dieser Verpflichtung erfolge. Auch werde der Bürge ausreichend durch die Pfändungsfreigrenzen der $\S \S 859$ f. ZPO geschützt. ${ }^{67}$

63 BGH, Urt v. 26.4.1972 (Az. IV ZR 18/71), NJW 1972, 1414, 1415 Rn. 16 als Verstoß gegen das Grundrecht auf Freizügigkeit gem. Art. 11 Abs. 1 GG.

64 BGH, Urt v. 17.4.1986 (Az. IX ZR 200/85), BGHZ 97, 372, 379 - Abrede über Gebrauch empfängnisverhütender Mittel.

65 Ausführlich Möllers, Juristische Methodenlehre, 2017, § 11 (im Erscheinen).

66 BVerfG, Urt. v. 6.2.2001 (Az. 1 BvR 12/92), BVerfGE 103, 89, 100 ff. - Unterhaltsverzicht: Verstoß gegen Art. 2 Abs. 1 in Verbindung mit Art. 6 Abs. 4 GG und Art. 6 Abs. 2 GG.

$67 \mathrm{Zu}$ früherer Rechtsprechung s. etwa BGH, Urt. v. 9.1.1989 (Az. IX ZR 124/88), BGHZ 106, 269, 272; BGH, Urt. v. 24.11.1992 (Az. XI ZR 98/92), BGHZ 120, 
Gegen diese Rechtsprechung ist massive Kritik erhoben worden, weil eine lebenslange Verschuldung das Streben nach Glück als unveräußerliches Menschenrecht verletzen würde. ${ }^{68}$ Honsell sprach vom ,eisigen Wind der Privatautonomie des ausgehenden 19. Jahrhunderts“, welche die Rechtsprechung des BGH begründen würde. ${ }^{69}$ Das BVerfG gab der Literatur Recht und stellte fest, dass der BGH fundamentale Grundrechte missachtet. Zwar lasse die Privatautonomie, wie sie in Art. 2 Abs. 1 GG garantiert ist, auch riskante Geschäfte zu, doch erfordere sie eine Selbstbestimmung, die bei einer ,gestörten Vertragsparität" - so der Terminus des BVerfG - nicht vorläge. Eine Korrektur sei immer dort erforderlich, wo wegen des Übergewichts einer Partei eine eigenverantwortliche Entscheidung nicht mehr vorhanden ist. ${ }^{70}$

Die heutige Rechtsprechung des BGH und die Vorgaben des BVerfG führen nicht dazu, dass jeder Vertrag einer Angemessenheitskontrolle unterzogen werden muss. Vielmehr sind die Vorgaben systemkonform durch eine strenge Anwendung des $\S 138$ Abs. 1 BGB durchzuführen. Wie so oft bei $§ 138$ BGB hat eine Gesamtwürdigung aller Umstände des Einzelfalls stattzufinden. ${ }^{71}$

\section{Verfassungskonforme Rechtsfortbildung}

\section{Die widersprüchliche Rechtsprechung des BVerfG}

a) Ablehnung einer verfasssungskonformen Rechtsfortbildung durch das BVerfG

Zum Teil wird die Rechtsfigur der verfassungskonformen Rechtsfortbildung abgelehnt. Eine Rechtsfortbildung soll unzulässig sein, wenn sie gegen den eindeutigen Wortlaut ${ }^{72}$ oder den subjektiven Willen des Gesetzgebers $^{73}$ verstößt. Ähnlich formuliert auch das BVerfG, dass die verfas-

272 ff.; Larenz/Canaris, Lehrbuch des Schuldrechts, Bd. 2, Hb. 2, 13. Aufl. 1994, $\S 80$. II, S. 9 f.

68 OLG Stuttgart, Urt. v. 12.1.1988 (Az. 6 U 86/87), NJW 1988, 833, 835.

69 Honsell, JZ 1989, 495 zu BGHZ 106, 269 ; kritisch auch Reinicke/Tiedtke, ZIP 1989, 613, 615.

70 BVerfG, Beschl. v. 19.10.1993 (Az. 1 BvR 567, 1044/89), BVerfGE 89, 214, $231 \mathrm{ff}$. - Bürgschaft vermögensloser Angehöriger.

71 Palandt/Ellenberger, BGB, 76. Aufl. 2017, § 138 Rn. $37 \mathrm{ff}$.

72 Zippelius, in: FG 25 Jahre BVerfG, Bd. II, 1976, S. 108, 115 f.

73 Stern, NJW 1958, 1435; Neuner, Privatrecht und Sozialstaat, 1998, S. 47 f.; Voßkuhle, AöR 125 (2000), 177, 197 f. 
sungskonforme Auslegung am „klar erkennbar geäußerten Willen des Gesetzgebers" ihre Grenze findet, weil sich das BVerfG ansonsten an die Stelle des demokratisch gewählten Gesetzgebers setzen würde. ${ }^{74}$

\section{b) Die verfassungskonforme Rechtsfortbildung als Rechtsfigur}

Auch wenn das Bundesverfassungsgericht nur den Begriff der „verfassungskonformen Interpretation" verwendet ${ }^{75}$ und formal eine verfassungskonforme Rechtsfortbildung ablehnt, hat es in zahlreichen Entscheidungen eine verfassungskonforme Rechtsfortbildung über die Wortlautgrenzen hinaus anerkannt. Zum Teil hat es sich sogar ausdrücklich gegen den damaligen Willen des Gesetzgebers entschieden und sich mit der objektiven Theorie auf das heutige Verständnis der Norm berufen. ${ }^{76}$ Die Rechtsprechung des BVerfG ist somit widersprüchlich. Konsequenterweise wird diese Rechtsfigur in der neueren Rechtsliteratur anerkannt. ${ }^{77}$ Allerdings sind die Vorgaben, wann eine solche Rechtsfortbildung unzulässig ist, zwischen den Senaten heftig umstritten. ${ }^{78}$

Grundsätzlich ist der Wille des Gesetzgebers zu beachten, gebietet dies doch der Respekt vor der gesetzgebenden Gewalt. ${ }^{79}$ Das BVerfG darf sich nicht zum Ersatzgesetzgeber aufschwingen. ${ }^{80}$ Sollten Gerichte ein formelles nachkonstitutionelles Bundesgesetz nach vorheriger, erfolgloser verfassungskonformer Auslegung für verfassungswidrig halten, müssen sie

74 BVerfG, Beschl. v. 14.10.2008 (Az. 1 BvR 2310/06), BVerfGE 122, 39, 60 f. Beratungshilfe: „Ein Normverständnis, das in Widerspruch zu dem klar erkennbar geäußerten Willen des Gesetzgebers, steht, kann auch im Wege der verfassungskonformen Auslegung nicht begründet werden“ unter Hinweis auf „BVerfGE 54, 277 [299 f.]; 71, 81 [105]; 90, 263 [275]; 95, 64 [93]; 98, 17 [45]; 99, 341 [358]; 101, 54 [86, 88] sowie 312 [329]; 112, 164 [183]“. S. auch Fn. 1.

75 S. oben Fn. 10.

76 BVerfG, Beschl. v. 27.3.1973, 1 BvR 112/65, BVerfGE 34, 269, 288 - Soraya.

77 Etwa Canaris, in: FS Honsell, 2004, S. 141, 155 ff.; Auer, in: Neuner, Grundrechte und Privatrecht aus rechtsvergleichender Sicht, 2007, S. 27, 30 ff.; Lembke, Einheit aus Erkenntnis?, 2009, S. 250 ff.

78 Voßkuhle, AöR 125 (2000), 177, 198.

79 S. oben Fn. 1.

80 Voßkuhle, AöR 125 (2000), 177, 198. Die von ihm angeführten BVerfG-Entscheidungen tragen die These allerdings nicht, da sie sich nur auf das Unterlassen gesetzgeberische Aktivität, nicht aber auf die verfassungskonforme Rechtsfortbildung beziehen. 
das BVerfG im Rahmen einer konkreten Normenkontrolle gem. Art. 100 Abs. 1 GG anrufen. Für Bürger besteht die Möglichkeit einer Verfassungsbeschwerde. ${ }^{81}$ Das BVerfG kann die Norm für verfassungswidrig und damit für nichtig erklären, $\S 78 \mathrm{~S} .1$ und $\S 95$ Abs. 3 S. 1 BVerfGG. Würde man in weitem Umfang eine verfassungskonforme Rechtsfortbildung gegen den Wortlaut des Gesetzes zulassen, würde die geltende Rechtslage zementiert und eine Lückenschließung durch förmliches Gesetz obsolet machen. ${ }^{82}$ So führte die Anerkennung des Persönlichkeitsrechtes durch das BVerfG dazu, dass bis heute der Gesetzgeber sich nicht genötigt sah ${ }^{83}$, eine gesetzliche Grundlage für dieses in der Praxis so wichtige Rechtsinstitut zu schaffen.

Eine zulässige Rechtsfortbildung muss sich deshalb in den gerade genannten Grenzen bewegen. In den folgenden Fallgruppen ist daher eine Rechtsfortbildung gegen den Wortlaut, die Systematik, den historischen Willen und den Telos der Norm (ausnahmsweise) zulässig, wenn eine eindeutige und klare Verletzung von Grundrechten vorliegt. Umgekehrt ist eine verfassungskonforme Rechtsfortbildung nicht mehr zulässig und damit contra legem, wenn sie massiv in Grundrechte Dritter eingreift.

\section{Fallgruppen zulässiger verfassungskonformer Rechtsfortbildung}

a) Teleologische Reduktion

Im Strafrecht und im Öffentlichen Recht lässt sich die verfassungskonforme Rechtsfortbildung regelmäßig als Ausdruck der Abwehrrechte-Dimension der Grundrechte beschreiben, weil hier der Staat in Grundrechtspositionen des betroffenen Bürgers eingreift. Am einfachsten lässt sich eine Rechtsfortbildung über den Wortlaut der Norm rechtfertigen, wenn sie eine teleologische Reduktion darstellt. Denn dann wird aus Gerechtigkeitsgründen Ungleiches ungleich behandelt, um dem Regelungszweck der Norm gerecht zu werden. ${ }^{84}$ Verfassungsrechtliche Rechtsfortbildung im Sinne einer teleologischen Reduktion ist hier zugunsten des betroffenen Bürgers möglich und fest etabliert. Maßgeblich sind unter anderem

81 Hillgruber, JZ 1996, 118, 122.

82 Hermes, VVDStRL 61 (2002), 119, 140.

83 S. unten Fn. 99.

84 Kramer, Juristische Methodenlehre, 4. Aufl. 2013, S. 225. 
das Recht auf freie Entfaltung der Persönlichkeit gem. Art. 2 Abs. 1 GG und die Justizgrundrechte gem. Artt. 101, 103 GG. Vor allem ist der Verhältnismäßigkeitsgrundsatz ${ }^{85} \mathrm{zu}$ beachten. $^{86}$

Das BVerfG hat gegen den klaren Wortlaut und den Willen ${ }^{87}$ des Gesetzgebers die lebenslange Freiheitsstrafe bei Mord gem. §211 StGB zugunsten des Täters teleologisch reduziert. Die Menschenwürde verlangt, dass der zu lebenslanger Freiheitsstrafe verurteilte Mörder die Chance haben muss, nach Verbüßung einer gewissen Strafzeit wieder in die Freiheit zu gelangen. ${ }^{88}$ Zugunsten des Täters wird das Mordmerkmal einschränkend ausgelegt, weil über den Wortlaut des $\S 211$ StGB hinaus verlangt wird, dass der Täter bei Heimtücke mit feindlicher Willensrichtung gehandelt hat. ${ }^{89}$

\section{b) Schutz vor offensichtlichen Grundrechtsverletzungen des Betroffenen}

aa) Deutlich komplizierter wird die Fragestellung allerdings, wenn man sich mit der verfassungskonformen Rechtsfortbildung über den damaligen (subjektiven) Willen des Gesetzgebers hinwegsetzen will. Die obige Formulierung des $\mathrm{BVerfG}^{90}$ scheint gegen eine solche Zulässigkeit zu sprechen. Das ist in dieser Absolutheit aber unrichtig, wie die Entscheidungen zeigen, in welchen sich das BVerfG selbst gegen den gesetzgeberischen Willen wandte. Am deutlichsten wird die verfassungskonforme Rechtsfortbildung heute immer noch mit der Entscheidung des BGH über den Schmerzensgeldanspruch bei Persönlichkeitsverletzung, in welcher der

85 Ausführlich Möllers, Juristische Methodenlehre, 2017, § 12 (im Erscheinen).

86 Für einen guten Überblick s. Kuhlen, Die verfassungskonforme Auslegung von Strafgesetzen, 2006; Dannecker, in: LK-StGB, 12. Aufl. 2007, § 1 Rn. 326-341.

87 S. hierzu die ausdrücklich für die lebenslange Freiheitsstrafe plädierenden Stimmen, wiedergegeben in BVerfG, Urt. v. 21.6.1977 (Az. 1 BvL 14/76), BVerfGE 45, 187, $194 \mathrm{ff} .-\S 211$ StGB.

88 BVerfG, Urt. v. 21.6.1977 (Az. 1 BvL 14/76), BVerfGE 45, 187, $258-\S 211$ StGB. Der Gesetzgeber hat mit den $\S \S 57 \mathrm{a}, 57 \mathrm{~b}$ StGB reagiert.

89 BGH, Urt. v. 22.9.1956 (Az. GSSt 1/56), BGHSt 9, 385, 390 - Heimtücke.

90 S. oben Fn. 1. Zudem BVerfG, Beschl. v. 11.6.1980 (Az. 1 PBvU 1/79), BVerfGE 54, 277, 299: „Nach der Rechtsprechung des Bundesverfassungsgerichts darf im Wege der Auslegung einem nach Wortlaut und Sinn eindeutigen Gesetz nicht ein entgegengesetzer Sinn verliehen, der normative Gehalt der auszulegenden Norm nicht grundlegend neu bestimmt, das gesetzgeberische Ziel nicht in einem wesentlichen Punkt verfehlt werden." 
BGH gegen Wortlaut und Willen des Gesetzgebers einen Schmerzensgeldsanspruch bei einer Persönlichkeitsrechtsverletzung entwickelte. In der Literatur wird diese Rechtsprechung als unzulässige Rechtsfortbildung contra legem kritisiert und gefordert, dass stattdessen das BVerfG die jeweils zu prüfende Norm hätte verwerfen müssen..$^{91}$ Tatsächlich geht es um den Streit zwischen objektiver und subjektiver Auslegung; ${ }^{92}$ in der Sora$y a$-Entscheidung hatte sich das BVerfG ausdrücklich zu einem Wertewandel und dem geänderten Verständnis der Normen bekannt. ${ }^{93}$

Im Herrenreiterfall war ohne Zustimmung mit dem Bild des Olympiasiegers Neckermann für ein Potenzmittel geworben worden; im Fernsehansagerin-Urteil wurde eine Fernsehansagerin u.a. als ,ausgemolkene Ziege" bezeichnet; im Soraya-Urteil wurde eine Interview mit der damaligen Prinzessin Soraya erfunden. Jeweils klagten die Betroffenen wegen einer Persönlichkeitsrechtsverletzung auf Schmerzensgeld.

Obwohl der Gesetzgeber bei der Schaffung des BGB ein absolutes Persönlichkeitsrecht nicht nach $\S 823$ Abs. 1 BGB schützen wollte, ${ }^{94}$ erkannte der BGH nach dem Zweiten Weltkrieg ein solches in einem ersten Schritt an. ${ }^{95}$ Und obwohl der Schmerzensgeldanspruch auf die gesetzlich geregelten Fälle begrenzt war ( $\$ 847$ BGB a.F.), entschied der BGH in einem zweiten Schritt, dass eine Verletzung dieses Rechts einen zivilrechtlichen Schmerzensgeldanspruch auslösen kann und begründete dies mit dem Per-

91 Konsequent kritisch zu dieser verfassungskonformen Rechtsfortbildung Larenz, Methodenlehre der Rechtswissenschaft, 6. Aufl. 1991, S. $426 \mathrm{ff}$;; Canaris, Die Feststellung von Lücken im Gesetz, 2. Aufl. 1983, S. 187 f.; Hillgruber, JZ 1996, 118, 119 ff.; Hermes, VVDStRL 61 (2002), 119, 131 f.; Neuner, Die Rechtsfindung contra legem, 2. Aufl. 2005, S. 130 f.; Rüthers/Fischer/Birk, Rechtstheorie, 8. Aufl. 2015, Rn. 806 ff.; Oeter, AöR 119 (1994), 529, 551 ff.; Diederichsen, Jura $1997,57,59 \mathrm{ff}$.

92 Zum Streitstand anschaulich etwa Enneccerus/Nipperdey, Allgemeiner Teil des Bürgerlichen Rechts, Bd. I/1, 15. Aufl. 1959, S. 54.II.; Engisch, Einführung in das juristische Denken, 11. Aufl. 2005, S. 160 ff.; Kramer, Juristische Methodenlehre, 4. Aufl. 2013, S. $121 \mathrm{ff}$; Fikentscher, Methoden des Rechts, Bd. III, 1976, S. 662 ff.; Fleischer, AcP 211 (2011), 318, 321 ff.

93 BVerfG, Beschl. v. 27.3.1973 (Az. 1 BvR 112/65), BVerfGE 34, 269, 288 - Soraya.

94 Protokolle zur 2. Lesung zum BGB, Bd. 2, 1897, S. 573 f., 640 f.; im Unterschied zum $§ 704$ Abs. 2 des 1. Entwurfs eines BGB. RG, Urt. v. 7.11.1908 (Az. I 638/07), RGZ 69, 401, 403 - Nietzsche-Briefe: „Ein allgemeines subjektives Persönlichkeitsrecht ist dem geltenden bürgerlichen Rechte fremd“; s. instruktiv Kötz/ Wagner, Deliktsrecht, 13. Aufl. 2016, $365 \mathrm{ff}$.

95 BGH, Urt. v. 25.5.1954 (Az. I ZR 211/53), BGHZ 13, 334, 338 - Schachtbrief. 
sönlichkeitsrecht des Artt. 1, 2 GG. ${ }^{96}$ Das BVerfG billigte diese „,verfassungskonforme Auslegung“. ${ }^{97}$ Die Verwendung des Begriffs „Auslegung“ ist unrichtig, handelt es sich hierbei doch um einen klaren Fall der verfassungskonformen Rechtsfortbildung, weil gegen Wortlaut, Willen, Systematik und Telos des BGB entschieden wurde.

Interessanterweise wird eine solche Begründung auch heute noch herangezogen: ${ }^{98}$ Obwohl der Gesetzgeber 2001 den $\S 847$ BGB a.F. strich, wurde auch in dem neu gefassten $\S 253$ BGB das allgemeine Persönlichkeitsrecht nicht in den Tatbestand aufgenommen. In den Bundestagsprotokollen beließ es der Gesetzgeber bei dem Hinweis, dass die Rechtsprechung das allgemeine Persönlichkeitsrecht anerkannt habe und man zum jetzigen Zeitpunkt nicht in der Lage sei, das Persönlichkeitsrecht umfassend gesetzlich zu regeln. ${ }^{99}$

bb) Ein Wandel der Wertvorstellungen allein kann für sich genommen nicht ausreichen, weil damit dem subjektiven Vorverständnis des jeweiligen Rechtsanwenders ${ }^{100}$ Tür und Tor geöffnet wäre. Eine Rechtsfortbildung gegen den Willen des damaligen Gesetzgebers wird man nur begründen können, wenn sich eine Rechtsschutzlücke besonders aufdrängt und eine Grundrechtsverletzung des Rechtssuchenden besonders offensichlich ist. Ein solcher Schutz ist etwa bei personalen Rechten mit starkem Bezug zur Menschenwürde gegeben, ${ }^{101}$ so dass Rechtsschutzlücken im geschriebenen Recht durch Rechtsfortbildung geschlossen werden können. Solche Schutzlücken hatte das BVerfG folglich bei der Persönlichkeitsrechtsver-

96 BGH, Urt. v. 14.2.1958 (Az. I ZR 151/56), BGHZ 26, 349, 354 - Herrenreiter; BGH, Urt. v. 5.3.1963 (Az. VI ZR 55/62), BGHZ 39, 124, 130 ff. - Fernsehansagerin. Im Ergebnis zustimmend etwa Larenz/Canaris, Schuldrecht, Lehrbuch des Besonderen Teils, Bd. 2, Hb. 2, 13. Aufl. 1994, § 80 I., S. 494.

97 BVerfG, Beschl. v. 14.2.1973 (Az. 1 BvR 112/65), BVerfGE 34, 269, 289 ff. Soraya.

98 BGH, Urt. v. 17.12.2013 (Az. VI ZR 211/12), BGHZ 199, 237 Rn. 40: „Schutzauftrag aus Art. 1 und 2 Abs. 1 GG“ - Geldentschädigung wegen Internetveröffentlichung; Palandt/Grüneberg, BGB, 76. Aufl. 2017, § 253 Rn. 10.

99 BT-Drs. 14/7752, S. 25.

100 Esser, Vorverständnis und Methodenwahl in der Rechtsfindung, 1970, S. 139 ff., $149 \mathrm{ff}$.

101 Neuner, JöR 59 (2011), S. 29, 38. 
letzung, ${ }^{102}$ im Volkszählungsurteil ${ }^{103}$ und in der Entscheidung zur OnlineDurchsuchung ${ }^{104}$ formuliert. ${ }^{105}$ In diesen Extremfällen erscheint es auch überzeugend, von einer Schutzpflicht des Staates ${ }^{106}$ oder vielleicht sogar von einer unmittelbaren Drittwirkung der Grundrechte ${ }^{107} \mathrm{zu}$ sprechen, um den Betroffenen vor der Rechtsverletzung zu schützen oder ihm zumindest eine Kompensation zu gewähren. Die Rechtsprechung kommt mit der Rechtsfortbildung ihrer verfassungsrechtlichen Pflicht gegenüber dem Bürger nach. Die Anerkennung des Persönlichkeitsrechts kann ebenfalls unschwer mit dem erforderlichen Grundrechtschutz nach Artt. 1, 2 Abs. 1 GG gerechtfertigt werden. ${ }^{108}$

102 BVerfG, Beschl. v. 27.3.1973 (Az. 1 BvR 112/65), BVerfGE 34, 269, 281 Soraya: „die Rechtsfigur des allgemeinen Persönlichkeitsrechts (...) füllt Lücken im Persönlichkeitsschutz aus, die hier trotz Anerkennung einzelner Persönlichkeitsrechte verblieben und im Laufe der Zeit aus verschiedenen Gründen immer fühlbarer geworden waren."

103 BVerfG, Urt. v. 15.12.1983 (Az. 1 BvR 209, 269, 362, 420, 440, 484/83), BVerfGE 65, 1, 42 f. - Volkszählungsurteil: „Damit haben sich in einer bisher unbekannten Weise die Möglichkeiten einer Einsicht- und Einflußnahme erweitert, welche auf das Verhalten des Einzelnen schon durch den psychischen Druck öffentlicher Anteilnahme einzuwirken vermögen."

104 BVerfG, Urt. v. 27.2.2008 (Az. 1 BvR 370, 595/07), BVerfGE 120, 274, 303 ff. Online-Durchsuchung: „Einer solchen lückenschließenden Gewährleistung [durch das allgemeine Persönlichkeitsrecht] bedarf es insbesondere, um neuartigen Gefährdungen zu begegnen, zu denen es im Zuge des wissenschaftlich-technischen Fortschritts und gewandelter Lebensverhältnisse kommen kann (vgl. BVerfGE 54, 148 [153]; 65, 1 [41]; 118, 168 [183])“.

$105 \mathrm{Zu}$ dieser Analyse, s. Hornung, Grundrechtsinnovationen, 2015, S. 355 ff.

106 S. oben Fn. 98.

107 Allgemein zur Lehre von der unmittelbaren Drittwirkung, begründet von Nipperdey, RdA 1950, 121, 125; ders., Allgemeiner Teil des Bürgerlichen Rechts, Bd. 1, 1959, S. 93 ff.; ihm folgend BAG, Urt. v. 3.12 .1954 (Az. 1 AzR 150/54), BAGE 1, 185, 193 f. Rn. 25; eine unmittelbare Drittwirkung von Art. 1 und 2 GG für das allgemeine Persönlichkeitsrecht wurde dann aber abgelehnt vom BAG, Beschl. v. 27.2.1985 (GS 1/84), BAGE 48, 122, 138 f. Rn. 45 - Weiterbeschäftigungsanspruch.

108 BVerfG, Beschl. v. 27.3.1973 (Az. 1 BvR 112/65), BVerfGE 34, 269, 281 f. Soraya sowie Fn. 98 f. A.A. Bruns, JZ 2014, 162, 165, der das BVerfG so versteht, als habe es die Rechtsfortbildung nur toleriert, ohne dass aber ein Grundrechsschutz erforderlich gewesen sei. 
3. Grenzen der Rechtsfortbildung durch die Verfassung (unzulässige Rechtsfortbildung contra legem)

a) Grenzen der Rechtsfortbildung im Strafrecht: das Gesetzlichkeitsprinzip

Eine unzulässige verfassungskonforme Rechtsfortbildung contra legem gibt es nicht, weil die Verfassungskonformität nicht verfassungswidrig sein kann. ${ }^{109}$ Allerdings gibt es Grenzen, wann eine Rechtsfortbildung noch verfassungskonform ist. Im Strafrecht ist dies bei Verstößen gegen die Wortlautgrenze der Fall, weil dann der betroffene Bürger die belastende Regelungsanordung nicht mehr wahrnehmen kann. Gleiches gilt, wenn die Vorschrift nicht bestimmt genug ist. ${ }^{110}$ Eine verfassungskonforme Rechtsfortbildung zugunsten des Bürgers ist aber denkbar. ${ }^{111}$

b) Grundrechtsbeeinträchtigung Dritter als relevante Grenze zulässiger Rechtsfortbildung? - der Streit der Verfassungssenate

Schmerzensgeldansprüche bei falschen Interviews schützen das Persönlichkeitsrecht, beeinträchtigen aber die Pressefreiheit. Der Schutz des Grundrechts einer Seite beeinträchtigt im Zweifel das Grundrecht der anderen Seite. ${ }^{112}$ Zwischen den beiden Senaten des BVerfG ist nun umstritten, inwieweit eine Rechtsfortbildung unzulässig ist, wenn Rechtspositionen bzw. Grundrechte Dritter betroffen sind. Drei Ansichten (aa, bb und c) lassen sich vertreten:

109 Auer, in: Neuner, Grundrechte und Privatrecht aus rechtsvergleichender Sicht, 2007, S. 27, 44.

110 BVerfG, Beschl. v. 21.6.1977 (Az. 2 BvR 308/77), BVerfGE 45, 363, 371 f.; s. Roxin, Strafrecht Allgemeiner Teil, Bd. 1, 4. Aufl. 2006, § 5 Rn. 11. Kuhlen, Die verfassungskonforme Auslegung von Strafgesetzen, 2006, S. 86 ff.; Dannecker, in: LK-StGB, 12. Aufl. 2007, § 1 Rn. 337.

111 Vgl. etwa BFH, Urt. v. 18.6.2009 (Az. VI R 14/07), BFHE 225, 393 - Aufwendungen bei Erststudium.

112 BVerfG, Beschl. v. 24.2.2015 (Az. 1 BvR 472/14), BVerfGE 138, 377, 392 f. Rn. 42 - Aufklärungsanspruch des Scheinvaters. 
aa) In der Entscheidung zur Rügeverkümmerung ${ }^{113}$ hatte der zweite Senat des BVerfG mit seiner Mehrheit formuliert, dass die Grenzen der Rechtsfortbildung unabhängig davon zu ziehen sind, ob die jeweilige Auslegung sich zugunsten oder zulasten betroffener Einzelner auswirkt. ${ }^{114}$

bb) Im Gegensatz dazu hatten drei Richter in einem abweichenden Sondervotum gerade umgekehrt formuliert: Die Grenzen der richterlichen Rechtsfortbildung verlangen gerade dort besondere Beachtung, wo sich die rechtliche Situation des Bürgers verschlechtert, ohne dass verfassungsrechtliche Gründe dafür ins Feld geführt werden können. ${ }^{15}$ Allgemein sollen die Grenzen bei Unterstützung von verfassungsmäßigen Rechten weiter und bei Verkürzung von Rechtspositionen enger anzusiedeln sein. ${ }^{116}$ Der erste Senat nahm die Überlegungen des Sondervotums nun auf und präzisierte diese weiter und stellte abstrakt folgende Regel auf:

„Belastet ein Zivilgericht eine Person etwa mit einer im Wege der Rechtsfortbildung begründeten Pflicht, so erfolgt dies zumeist, um die Rechtsposition einer anderen Person zu stärken. Je schwerer der verfassungsrechtliche Gehalt der gestärkten Position wiegt, umso klarer ist eine entsprechende Lösung dem Gericht wie dem Gesetzgeber durch die Verfassung vorgezeichnet und umso weiter kann die Befugnis der Gerichte reichen, diese Position im Wege der Rechtsfortbildung - auch unter Belastung einer gegenläufigen, aber schwächeren Rechtsposition - durchzusetzen (so etwa BVerfGE 96, 56 [62ff.]). Umgekehrt gilt jedoch genauso: Je schwerer die Belastung verfassungsrechtlich wiegt und je schwächer der verfassungsrechtliche Gehalt der damit durchzusetzenden Gegenposition ist, umso enger sind die Grenzen für die Rechtsfortbildung gesteckt, umso strikter muss sich also die zivilgerichtliche

113 Von einer Rügeverkümmerung spricht man dann, wenn im Rahmen einer Revision formelle Fehler des Verfahrens gerügt werden, die zwar im Protokoll enthalten sind, aber aufgrund nachträglicher Berichtigung des Protokolls wieder weggefallen sind und damit zur Unbegründetheit der Revision führen.

114 BVerfG, Beschl. v. 15.1.2009 (Az. 2 BvR 2044/07), BVerfGE 122, 248, 268 Rn. 60 - Rügeverkümmerung: „Die Grenzen, die sich aus Art. 20 Abs. 2 Satz 2 und Abs. 3 GG für die richterliche Auslegung des einfachen Rechts ergeben, können daher nicht prinzipiell enger oder weiter gesteckt sein je nachdem, ob die jeweilige Auslegung sich zugunsten oder zu Lasten betroffener Einzelner auswirkt.“

115 Sondervotum der Richter Voßkuhle, Osterloh und Di Fabio: BVerfG, Beschl. v. 15.1.2009 (Az. 2 BvR 2044/07), BVerfGE 122, 248, 282, 301 Rn. 144 - Rügeverkümmerung.

116 Sondervotum der Richter Voßkuhle, Osterloh und Di Fabio: BVerfG, Beschl. v. 15.1.2009 (Az. 2 BvR 2044/07), BVerfGE 122, 248, 282, 286 Rn. 105 - Rügeverkümmerung; zustimmend BVerfG, Beschl. v. 24.2.2015 (Az. 1 BvR 472/14), BVerfGE 138, 377, 392 Rn. 41 - Aufklärungsanspruch des Scheinvaters. 
Rechtsfindung innerhalb der Grenzen des gesetzten Rechts halten. Die Grenzen richterlicher Rechtsfindung verlangen gerade dort besondere Beachtung, wo sich die rechtliche Situation des Bürgers verschlechtert, ohne dass verfassungsrechtliche Gründe dafür ins Feld geführt werden können (BVerfGE 122, 248 [301] - abw. M.). Auf eine privatrechtliche Generalklausel lässt sich eine verfassungsrechtlich schwerwiegende Belastung eines Beteiligten dann umso weniger stützen, je weniger sich im einfachgesetzlichen Umfeld Anknüpfungspunkte dafür finden lassen (vgl. Röthel, Normkonkretisierung im Privatrecht, 2004, S. 120f.).“117

c) Eigene Ansicht zur Rechtsfortbildung contra legem bei schwerer Grundrechtsbeeinträchtigung Dritter

aa) Die Ansicht der Mehrheit des zweiten Senats, dass Grundrechte überhaupt keine Rolle für die Grenzen zulässiger Rechtsfortbildung spielen, ist zu eng. Wenn sich der zu Schützende auf Grundrechte berufen kann, um die klassischen Auslegungsfiguren zu überspielen, dann muss dieses Recht auch der anderen Seite zustehen. Mit anderen Worten: die eindeutige und klare Verletzung von Grundrechten muss eine Rechtsfortbildung begründen und damit den Wortlaut des einfachen Rechts überspielen können, sodass etwa die verfassungskonforme Rechtsfortbildung zulässig ist. Und spiegelbildlich dazu ist eine verfassungskonforme Rechtsfortbildung dann unzulässig, wenn sie massiv in Grundrechte Dritter eingreift.

Damit war eine rechtsfortbildende Einschränkung der deliktischen Haftung des Sachverständigen auf grobe Fahrlässigkeit unzulässig, weil das fehlerhafte Gutachten dazu führte, dass der Strafgefangene nicht aus der psychiatrischen Anstalt entlassen wurde und eine solche Freiheitsberaubung massiv in Grundrechte eingreift. Im Unterschied zur Soraya-Entscheidung wurde durch die Rechtsfortbildung die Position der betroffenen Gegenseite (Strafgefangener) deutlich verschlechtert. ${ }^{118}$ Der Gesetzgeber hat mit der Einführung des

117 BVerfG, Beschl. v. 24.2.2015 (Az. 1 BvR 472/14), BVerfGE 138, 377, 392 ff. Rn. 42 ff. - Aufklärungsanspruch des Scheinvaters.

118 Die Rechtsfortbildung würde zwar die Rechtsposition des Sachverständigen, der sich auf Art. 12 Abs. 1 GG berufen kann, verbessern. Diese Überlegung hat allerdings zurückzutreten. 
$\S 839 \mathrm{a} \mathrm{BGB}^{119}$ reagiert und die Haftung auf Vermögensschäden erweitert, aber auf grobe Fahrlässigkeit und Vorsatz beschränkt. ${ }^{120}$

bb) Umgekehrt sind die Ansicht des ersten Senats und das Sondervotum des zweiten Senats, die immer eine Grundrechtsabwägung zwischen beiden Parteien vornehmen, viel zu weit. Das BVerfG gerierte zur Superrevisionsinstanz, weil jeder Streit um zivilrechtliche Interessen nun problemlos grundrechtlich ,aufgeladen“ werden kann. Bekanntlich haben die Fachgerichte aber grundsätzlich die bessere Expertise, die Sachprobleme zu entscheiden. Formal fällt auf, dass der erste Senat komparative Sätze im Sinne von „Je (...) desto“ formuliert. Solche Sätze sind, wie beim Beweglichen System, wenig eindeutig und beeinträchtigen die Rechtssicherheit. ${ }^{121}$ Inhaltlich erinnert die Prüfung der Grundrechte an eine verfassungsorientierte Auslegung, die aber nicht dazu in der Lage ist, ein eindeutiges Ergebnis zu bestimmen, sondern nur ein Argument einer Abwägung darstellt. Zudem fällt auf, dass das Grundgesetz keine Grenze kennt, wonach Grundrechtsbeeinträchtigungen Dritter eine Grenze zulässiger Rechtsfortbildung begründen. Es lässt sich auch kaum eine verlässliche Grenze ausmachen, weil beiden Seiten Grundrechte zustehen. ${ }^{122}$ Die Problematik wurde vom BVerfG an dem Auskunftsanspruch des Scheinvaters gegenüber der Mutter thematisiert.

Auskunftsanspruch des Scheinvaters: Die Zivilgerichte haben einen Auskunftsanspruch des Scheinvater gegenüber der Mutter aus $\S 242$ BGB bejaht, um zu verhindern, dass er statt des wirklichen Vaters über Jahre hinweg den Kindesunterhalt zahlen muss. ${ }^{123}$ Dagegen hat das BVerfG eine solche Rechtsfortbildung für rechtlich unzulässig erklärt: Ein Auskunftsanpruch würde das Persönlichkeitsrecht der Mutter und des leiblichen Vaters verletzen, weil sie dann Auskunft über ihre sexuellen Kontakte geben müssten. ${ }^{124}$ Das BVerfG

119 BT-Drucks. 13/10435, S. 18; BT-Drucks. 14/7752, S. 28.

120 BVerfG v. 11.10.1978 (Az. 1 BvR 84/74), BVerfGE 49, 304, 320 - Sachverständigenhaftung. A.A. noch BGH, Urt. v. 8.5.1956 (Az. VI ZR 113/71), BGHZ 62, 54, 56 - Sachverständigenhaftung.

121 Ähnlich auch Neuner, JZ 2016, 435, 439: ,äußerst subtile und höchst schwierige verfassungssrechtliche Erwägungen seitens der Zivilgerichte.“

122 Neuner, JZ 2016, 435, 439.

123 BGH, Urt. v. 9.11.2011 (Az. XII ZR 136/09), BGHZ 191, 259 - Auskunftsanspruch des Scheinvaters; BGH, Urt. v. 20.2.2013 (Az. XII ZB 412/11), BGHZ 196, 207 - Auskunftsanspruch des Scheinvaters.

124 Rauscher, JZ 2015, 624, 625. 
sieht die Interessen der Mutter generell höherrangig gegenüber dem rein „einfachgesetzlichen Regreßanspruch" des Scheinvaters an. ${ }^{125}$

Die Entscheidung des BVerfG ist massiv kritisiert worden. Bereits die abstrakte Höhergewichtung der Interessen der Mutter gegenüber denen des Scheinvaters ist höchst angreifbar. Für die Interessen des Scheinvaters spricht schon der durch Art. 6 Abs. 1 GG vorgegebene Schutz der Ehe, den das BVerfG vollständig ausblendet. ${ }^{126}$ Der Regressanspruch des Scheinvaters gegenüber dem leiblichen Vater liefe faktisch leer, wenn man einen Auskunftsanspruch ablehnt; der Scheinvater würde rechtsschutzlos gestellt. ${ }^{127}$ Wenn aber das Kind unstreitig gegen seinen leiblichen Vater einen verfassungsrechtlich abgesicherten Auskunftsanspruch besitzt, ${ }^{128}$ sollte man einen solchen Auskunftsanspruch auch für den Scheinvater bejahen und damit beide Fälle gleich behandeln. ${ }^{129}$ Wenn man einen solchen Anspruch aber ablehnt, würde das Kind in die Auseinandersetzung miteinbezogen, da der Scheinvater das Kind nun drängen wird, Auskunft über den leiblichen Vater zu erteilen. ${ }^{130}$ Eine solche Folge zulasten des Kindeswohls ist aber zu vermeiden.

Schließlich ist auch zweifelhaft, ob die Mutter überhaupt schutzwürdiges Vertrauen besitzt. Darf die Mutter mithin dem Scheinvater die Identität des leiblichen Vaters verheimlichen und ihn damit dauerhaft mit Unterhaltszahlungen belasten, die der leibliche Vater zu zahlen hat? Dagegen spricht schon, dass sie die Vermögensbelastung hervorruft, weil sie das Kind mit einem Dritten gezeugt hat und nun den Unterhaltsanspruch des Scheinvaters auslöst. ${ }^{131} \mathrm{Ihr}$ eigenes treuwidriges Verhalten löst die Unterhaltszahlungen aus; sie könnte den Scheinvater über die Vaterschaft des Dritten aufklären, bevor Unterhaltsansprüche relevant werden. ${ }^{132}$ Der Scheinvater ist gegenüber der Mutter in einer Situation struktureller Benachteiligung, ${ }^{133}$ weil die Problematik der Scheinmutter biologisch nicht möglich ist und nur sie den leiblichen Vater kennt. Das BVerfG verweigert sich einer interessengerechten Lösung, indem es der treuwidrigen Mutter ermöglicht, den leiblichen Vater zu schützen, in-

125 BVerfG, Beschl. v. 24.2.2015 (1 BvR 472/14), BVerfGE 138, 377, 394 Rn. 46 Auskunftsanspruch des Scheinvaters.

126 Kritisch folglich Forschner, FuR 2015, 451, 453.

127 Preu $\beta$, NJW 2015, 1509, 1510, Muckel, JA 2015, 953, 954.

128 BVerfG, Beschl. v. 6.5.1997 (Az. 1 BvR 409/90), BVerfGE 96, 56, 61 f. - Auskunftsanspruch des Kindes gegen den Vater: „Mit der Herleitung eines Auskunftsanspruchs gegen die Mutter aus $\S 1618$ a BGB hat das Landgericht nicht die Grenzen unzulässiger Rechtsfortbildung überschritten."

129 Neuner, JZ 2016, 435, 438.

130 Löhnig, NZFam 2015, 355, 359.

131 Sachs, JuS 2015, 860, 861.

132 Rauscher, JZ 2015, 624, 626.

133 Rauscher, JZ 2015, 624, 626. 
dem der Gehörnte Alimente für das Kind zu zahlen hat. ${ }^{134}$ Damit wird eine Rechtsschutzlücke perpetuiert.

cc) Im Gegensatz zur Ansicht des ersten Senats kann somit nicht schon jede Grundrechtsbeeinträchtigung eines Dritten eine Rechtsfortbildung blockieren. Vielmehr sollte eine verfassungsrechtliche Kontrolle durch das BVerfG erst eingreifen, wenn ein Grundrecht des Dritten eindeutig und schwer verletzt ist. Plastisch wird man einen Verstoß gegen die Verfassung etwa dann bejahen können, wenn auch ein gleichlautendes Gesetz nicht mehr mit der Verfassung vereinbar wäre. ${ }^{135}$

\section{Fazit}

Zusammenfassend kann bei der Auslegung einfachgesetzlicher Normen die Verfassung in dreierlei Hinsicht Beachtung finden.

1. Zum einen ist die Werteordnung der Verfassung neben dem klassischen Viererkanon der Auslegungsmethoden in Form der verfassungsorientierten Auslegung ein zusätzlicher und gleichberechtigter Abwägungsmaßstab. Die Ausstrahlungswirkung der Verfassung kommt somit schon als Erkenntnisquelle beim Normverständnis zum Tragen und garantiert eine Optimierung im Sinne der Verfassung.

2. Zum anderen werden in einem zweiten Schritt über die verfassungskonforme Auslegung solche Auslegungsergebnisse zwingend ausgeschieden, die nicht mit der Verfassung vereinbar sind. Insoweit kommt der Vorrang der Verfassung gegenüber dem einfachen Recht zur Geltung.

3. Wenn nach diesen beiden Schritten kein verfassungskonformes Ergebnis vorliegt, ist die Norm vom BVerfG grundsätzlich als verfassungswidrig zu verwerfen. Ausnahmsweise kann sich das BVerfG im Wege einer verfassungskonformen Rechtsfortbildung über den Wortlaut und Willen des historischen Gesetzgebers hinwegsetzen, wenn nur so schwer-

134 Deutlich Löhnig; NZFam 2015, 355, 359: es komme darauf an, ob die Mutter den biologischen Vater „in die Pfanne hauen“ oder schützen wolle.

135 Zur sog. Schumann'schen Formel s. Schumann, Verfassungs- und Menschenrechtsbeschwerde gegen richterliche Entscheidungen, 1963, S. 239; BVerfG, Urt. v. 14.2.1989 (Az. 1 BvR 1131/87), BVerfGE 79, 283, 290; BVerfG, Beschl. v. 3.4.1990 (Az. 1 BvR 1186/89), BVerfGE 82, 6, 15 f.; BverfG, Beschl. v. 26.6.1991 (Az. 1 BvR 779/85), BVerfGE 84, 212, 228 f. - Arbeitskampfrecht; Söllner, ZG 1995, 1, 14 f. 
wiegende Grundrechtsverletzungen des Betroffenen verhindert werden können. Eine Grenze der Rechtsfortbildung ist hingegen erreicht, wenn hierdurch die Grundrechte Dritter massiv beeinträchtigt würden. 